\title{
ON DEFINING EQUATIONS FOR THE JACOBIAN LOCUS IN GENUS FIVE ${ }^{1}$
}

\author{
ROBERT D. M. ACCOLA ${ }^{2}$
}

\begin{abstract}
In the space of principally polarized abelian varieties of dimension 5 , eight special theta relations can be chosen to define eight hypersurfaces whose intersection contains the Jacobian locus as a component.
\end{abstract}

Let $\delta_{5}$ be the Siegel generalized upper half plane of principally polarized abelian varieties of dimension 5. Let $\mathcal{g}_{5}$ be the Jacobian locus and $\mathcal{H}_{5}$ the hyperelliptic sublocus of $\mathscr{g}_{5}$. Then $\mathcal{H}_{5} \subset \mathcal{g}_{5} \subset \mathcal{S}_{5}$ with respective dimensions 9,12 , and 15. From the Schottky-Jung-Farkas-Rauch theorem [5, p. 212] one can derive equations in thetanulls, the special theta relations, which hold for all $A \in \mathcal{G}_{5}$ but not for all $A \in \mathcal{S}_{5}$. These special theta relations therefore define hypersurfaces in $\mathcal{S}_{5}$, each of which contains $\mathcal{G}_{5}$. In this paper we show that the intersection of these hypersurfaces contains $g_{5}$ as a component; that is, these special theta relations give, locally, defining equations for $\mathscr{G}_{5}$ in $\delta_{5}$.

A recent result of $\mathrm{D}$. Mumford is an essential ingredient of the proof. Otherwise we use methods that go back to $M$. Noether [4]. We must presuppose some acquaintance with the classical theory of theta functions. As references we give [5], [2, Chapter 7] and also [1] for a very brief resumé. We will briefly introduce some of the necessary ideas for dimension 5, hoping they are already somewhat familiar to the reader. Our notation will follow [2].

Let $(\pi i E, B)$ be a $5 \times 10$ period matrix for an abelian variety $A \in \mathcal{S}_{5}$. For $u \in C^{5}$ let $\theta[\varepsilon](u ; B)$ be a first order theta function with theta characteristic (Th. Char.) $[\varepsilon]$. $[\varepsilon]$ is called an even (odd) Th. Char. if this function is even (odd), and this is denoted $|\varepsilon|=1(|\varepsilon|=-1)$. If $|\varepsilon|=1$ we call $\theta[\varepsilon](0 ; B)$ a thetanull and write it simply as $\theta[\varepsilon]$. A set of even theta characteristics $\left\{\left[\varepsilon_{i}\right]\right\}$ will be called azygetic if $\left|\varepsilon_{i} \varepsilon_{j} \varepsilon_{k}\right|=-1$ for 3 different Th. Char.'s and if the sum of an even number is not the zero period characteristic (Per. Char.), except possibly for the sum of all the $\left[\varepsilon_{i}\right]$. For dimension 5 an azygetic set of eleven even Th. Char.'s $\left\{\left[\varepsilon_{i}\right]\right\}$ together with $[n]\left(=\left[\sum_{i=1}^{11} \varepsilon_{i}\right]\right)$ will be called a hyperelliptic fundamental system of theta characteristics (H.F.S. of Th. Char.'s). In this case $|n|=-1$ and $\left|n \varepsilon_{i} \varepsilon_{j}\right|=1$ if $i \neq j$. If we denote the Per. Char.

Received by the editors December 18, 1981.

1980 Mathematics Subject Classification. Primary 30F10; Secondary 14H15.

Key words and phrases. Riemann surface, theta function, abelian variety.

'The author thanks Professors Joseph Harris and Alan Landman for valuable discussions concerning the material in this paper.

${ }^{2}$ Research supported by the National Science Foundation. 
$\left(n \varepsilon_{i}\right)$ by $\left(a_{i}\right)$ then $\left[\varepsilon_{i}\right]=\left[n a_{i}\right]$ and $\left[n \varepsilon_{i} \varepsilon_{j}\right]=\left[n a_{i} a_{j}\right]$. Write $\left[n \Sigma^{s} a\right]$ for a Th. Char. where $\Sigma^{s} a$ stands for a sum of $s$ different $a$ 's. Then $\left[n \Sigma^{s} a\right]$ is even if $s=1,2,5,6,9,10$ and odd if $s=0,3,4,7,8,11$.

Let $W_{5}$ be a hyperelliptic Riemann surface of genus 5. By choosing a suitable canonical homology basis on $W_{5}[2$, p. 445] one can find a H.F.S. of Th. Char.'s so that $\theta\left[n a_{i}\right]=\theta\left[n a_{i} a_{j}\right]=0$ for all $i, j$ and $\theta\left[n \Sigma^{5} a\right] \neq 0$ for all Th. Char. of this form. (Since $\left(\sum_{i=1}^{11} a_{i}\right)=(0)$ it follows that $\theta\left[n \Sigma^{6} a\right] \neq 0$ also.)

General theta relations are homogeneous polynomials in the thetanulls which are zero for all $A \in \mathcal{S}_{5}$. We now describe those general theta relations for dimension 5 which we will need [4].

Suppose $\left[\varepsilon_{1}\right], \ldots,\left[\varepsilon_{6}\right]$ is an azygetic set of six even Th. Char.'s and $G_{4}$ is a group of four Per. Char.'s so that (i) $\left|\varepsilon_{i} \sigma\right|=1$ for $i=1,2, \ldots, 6$ and all $(\sigma) \in G_{4}$, and (ii) $\left(\sum_{i=1}^{6} \varepsilon_{i}\right) \in G_{4}$. Let

$$
q_{i}=\prod_{(\sigma) \in G_{4}} \theta\left[\varepsilon_{i} \sigma\right]
$$

Then

$(\mathrm{GR})_{1}$

$$
\sum_{i=1}^{6} \pm q_{i}=0
$$

Suppose $\left[\varepsilon_{1}\right], \ldots,\left[\varepsilon_{4}\right]$ is an azygetic set of four even Th. Char.'s and $G_{8}$ is a group of 8 Per. Char.'s so that $\left|\varepsilon_{i} \sigma\right|=1$ for $i=1, \ldots, 4$ and for all $(\sigma) \in G_{8}$. Let $G_{4}$ be a subgroup of $G_{8}$ of order four and let $(\tau) \in G_{8}-G_{4}$. Let

$$
q_{i}=\prod_{(\sigma) \in G_{4}} \theta\left[\varepsilon_{i} \sigma\right] \text { and } q_{i}^{\prime}=\prod_{(\sigma) \in G_{4}} \theta\left[\varepsilon_{i} \sigma \tau\right] \text {. }
$$

Then

$(\mathrm{GR})_{2}$

$$
\sum_{i=1}^{4}\left( \pm q_{i} \pm q_{i}^{\prime}\right)=0 \text {. }
$$

As an application of (GR $)_{2}$ for a H.F.S. of Th. Char.'s let $\left[\varepsilon_{i}\right]=\left[n a_{i}\right], i=1,2,3,4$. Let $(\tau)=\left(a_{5}\right)$ and

$$
G_{4}=\left\{(0),\left(a_{6} a_{7} a_{8} a_{9}\right),\left(a_{6} a_{7} a_{10} a_{11}\right),\left(a_{8} a_{9} a_{10} a_{11}\right)\right\} .
$$

Then

$$
q_{i}=\theta\left[n a_{i}\right] \theta\left[n a_{i} a_{6} a_{7} a_{8} a_{9}\right] \theta\left[n a_{i} a_{6} a_{7} a_{10} a_{11}\right] \theta\left[n a_{i} a_{8} a_{9} a_{10} a_{11}\right]
$$

and

$$
q_{i}^{\prime}=\theta\left[n a_{i} a_{5}\right] \theta\left[n a_{i} a_{5} a_{6} a_{7} a_{8} a_{9}\right] \theta\left[n a_{i} a_{5} a_{6} a_{7} a_{10} a_{11}\right] \theta\left[n a_{i} a_{5} a_{8} a_{9} a_{10} a_{11}\right] .
$$

Now by an arbitrary permutation of the digits from 1 to 11 we obtain the following lemma.

LemMa 1. Suppose for 5 distinct integers $i, j, k, l, m$ from 1 to 11 seven of the eight thetanulls with the following Th. Char.'s are zero: $\left[n a_{i}\right],\left[n a_{j}\right],\left[n a_{k}\right],\left[n a_{l}\right],\left[n a_{i} a_{m}\right]$, $\left[n a_{j} a_{m}\right],\left[n a_{k} a_{m}\right],\left[n a_{l} a_{m}\right]$. Suppose all $\theta\left[n \Sigma^{5} a\right] \neq 0$. Then the thetanull with the eighth Th. Char. is also zero. 
Using a recent result of D. Mumford we now give local defining equations for $\mathcal{H}_{5}$ in $\mathcal{S}_{5}$.

Lemma 2. Suppose $A \in \mathcal{S}_{5}$ is near $\mathcal{H}_{5}$ so that $\theta\left[n \Sigma^{5} a\right] \neq 0$ for all such Th. Char.'s with respect to a suitable H.F.S. of Th. Char.'s. Suppose $\theta\left[n a_{i}\right]=0$ for $i=1,2, \ldots, 11$. Then $A \in \mathcal{H}_{5}$.

Proof. We first systematically apply the general theta relations to show that $\theta\left[n a_{i} a_{j}\right]=0$ for $1 \leqslant i<j \leqslant 11$. First apply $(\mathrm{GR})_{1}$ to $\left[n a_{1}\right],\left[n a_{2}\right], \ldots,\left[n a_{5}\right],\left[n a_{6} a_{7}\right]$. Let

$$
G_{4}=\left\{(0),\left(a_{8} a_{9} a_{10} a_{11}\right),\left(a_{6} a_{7} a_{8} a_{9}\right),\left(a_{6} a_{7} a_{10} a_{11}\right)\right\}
$$

Then

$$
q_{6}=\theta\left[n a_{6} a_{7}\right] \theta\left[n a_{8} a_{9}\right] \theta\left[n a_{10} a_{11}\right] \theta\left[n a_{6} a_{7} a_{8} a_{9} a_{10} a_{11}\right]=0 .
$$

We conclude that for any 6 distinct integers from 1 to 11 ,

$$
\theta\left[n a_{i} a_{j}\right] \theta\left[n a_{k} a_{l}\right] \theta\left[n a_{m} a_{r}\right]=0 .
$$

Suppose $\theta\left[n a_{1} a_{2}\right] \neq 0$. Then $\theta\left[n a_{i} a_{j}\right] \theta\left[n a_{k} a_{l}\right]=0$ for $3 \leqslant i<j<k<l \leqslant 11$. Suppose now, in addition, that $\theta\left[n a_{3} a_{4}\right] \neq 0$. Then $\theta\left[n a_{k} a_{l}\right]=0$ for $5 \leqslant k<l \leqslant 11$. Now apply Lemma 1 to $\left[n a_{3}\right],\left[n a_{5}\right],\left[n a_{6}\right],\left[n a_{7}\right],\left[n a_{3} a_{8}\right],\left[n a_{5} a_{8}\right],\left[n a_{6} a_{8}\right],\left[n a_{7} a_{8}\right]$ to conclude that $\theta\left[n a_{3} a_{8}\right]=0$. Thus $\theta\left[n a_{3} a_{k}\right]=\theta\left[n a_{4} a_{k}\right]=0$ for $5 \leqslant k \leqslant 11$.

Now apply Lemma 1 to $\left[n a_{3}\right],\left[n a_{5}\right],\left[n a_{6}\right],\left[n a_{7}\right],\left[n a_{3} a_{4}\right],\left[n a_{4} a_{5}\right],\left[n a_{4} a_{6}\right]$, $\left[n a_{4} a_{7}\right]$ to conclude that $\theta\left[n a_{3} a_{4}\right]=0$ after all. Thus the hypothesis $\theta\left[n a_{1} a_{2}\right] \neq 0$ implies $\theta\left[n a_{k} a_{l}\right]=0$ for $3 \leqslant k<l \leqslant 11$. By an entirely analogous argument we conclude that $\theta\left[n a_{1} a_{2}\right]$ itself must be zero.

Thus the theta functions for $A$ have vanishing properties which mimic those of a hyperelliptic Jacobian. Mumford's theorem [3] states that such an $A$ must be in $\mathcal{H}_{5}$; that is, $\mathcal{H}_{5}$ is characterized by the vanishings of its theta functions with half-integer Th. Char.'s. Q.E.D.

We now consider the special theta relations for genus 5 , that is, equations in the thetanulls which hold on $y_{5}$ but not on $\bar{s}_{5}$.

Let $\left[\varepsilon_{1}\right], \ldots,\left[\varepsilon_{4}\right]$ be an azygetic set of four even Th. Char.'s. Let $G_{8}$ be a group of eight Per. Char.'s so that $\left|\varepsilon_{i} \sigma\right|=1$ for all $i$ and all $(\sigma) \in G_{8}$. Let

$$
r_{i}=\prod_{(\sigma) \in G_{8}} \theta\left[\varepsilon_{i} \sigma\right]
$$

Then

$$
\sum_{i=1}^{4} \pm \sqrt{r_{i}}=0
$$

or

$$
\left(\sum r_{i}^{2}-2 \sum r_{i} r_{j}\right)^{2}=64 r_{1} r_{2} r_{3} r_{4}
$$

We now show that eight special theta relations give local defining equations for $q_{5}$ in $\mathcal{S}_{5}$. Let $A_{0} \in \mathcal{H}_{5}$ and let $A\left(\varepsilon \mathcal{S}_{5}\right)$ be near $A_{0}$ so that $\theta\left[n \Sigma^{5} a\right] \neq 0$ for a suitable H.F.S. of Th. Char. Near $A_{0}, \mathcal{H}_{5}$ is defined by eleven equations $\theta\left[n a_{i}\right]=0$, 
$i=1,2, \ldots, 11$. We now write eleven equations locally defining $\mathcal{H}_{5}$ near $A_{0}$ in a different manner.

Apply formula (SR) letting $\left[\varepsilon_{i}\right]$ be $\left[n a_{1}\right],\left[n a_{2}\right],\left[n a_{3}\right],\left[n a_{4}\right]$ and letting

$$
G_{8}=\left\langle\left(a_{5} a_{6} a_{7} a_{8}\right),\left(a_{5} a_{6} a_{9} a_{10}\right),\left(a_{5} a_{7} a_{9} a_{11}\right)\right\rangle .
$$

Then $r_{i}$ is of the form $\theta\left[n a_{i}\right] \Pi^{7} \theta\left[n \Sigma^{5} a\right]$, where the last 7 factors of the products are not zero. Let $(\mathrm{SR})_{1234}$ be the special relation $\sum_{i=1}^{4} \pm \sqrt{r_{i}}=0$. Let $(\mathrm{SR})_{123 k}$ be the special relation where 4 and $k$ are transposed, $k=5,6, \ldots, 11$. Then the eleven equations

$$
\theta\left[n a_{1}\right]=\theta\left[n a_{2}\right]=\theta\left[n a_{3}\right]=0
$$

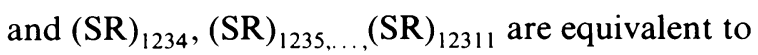

$$
\theta\left[n a_{i}\right]=0, \quad i=1, \ldots, 11, \text { near } A_{0} .
$$

Consequently, this new set of eleven equations locally defines $\mathcal{H}_{5}$ in $\mathcal{S}_{5}$.

The eight special theta relations $(\mathrm{SR})_{123 k}$ define eight hypersurfaces in $\mathcal{S}_{5}$ each containing $\mathscr{g}_{5}$. Let $V$ be an irreducible component of the intersection of these eight hypersurfaces which contains $\mathscr{G}_{5}$. The equations $\theta\left[n a_{i}\right]=0, i=1,2,3$, determine three hypersurfaces whose intersection with $V$ equals $\mathcal{H}_{5}$ by Lemma 2. Consequently, $V$ has codimension $\geqslant 3$. It follows that $V=g_{5}$ since $g_{5}$ has codimension 3 .

THEOREM. In $\mathcal{S}_{5}$ eight special theta relations can be chosen which give local defining equations for $\mathcal{G}_{5}$.

We conclude with some comments in the case of genus 6 . Let $\mathscr{P}_{7}$ be the principally polarized abelian varieties of dimension 6 which are Pryms of Riemann surfaces of genus 7. Then $\mathscr{f}_{6} \subset \mathscr{P}_{7} \subset \mathcal{S}_{6}$ where the dimensions are 15,18 , and 21 . One would like to show that the special theta relations for genus 6 are local defining equations for $q_{6}$ in $\delta_{6}$. The author has been unable to generalize the methods of this paper to this case. However, it can be shown that eight special relations as functions on $\mathcal{P}_{7}$ define $g_{6}$ locally. This is accomplished by specializing eight special relations for genus 6 to eight special relations for genus 5 known to define $q_{5}$ locally. The generalization of this latter type of result to arbitrary genus appears possible.

\section{REFERENCES}

1. Robert D. M. Accola, Some loci in Teichmüller space for genus six defined by vanishing thetanulls (submitted for publication).

2. A. Krazer, Lehrbuch der Thetafunktionen, Teubner, Leipzig, 1903 (Chelsea reprint).

3. D. Mumford, Tata lectures on theta, II, Birkhäuser, Boston, Mass. (to appear).

4. M. Noether, Zur Theorie der Thetafunctionen von beliebiq vielen Argumenten, Math. Ann. 16 (1880), pp. 270-344.

5. H. E. Rauch and H. M. Farkas, Theta functions with applications to Riemann surfaces, Williams and Williams, Baltimore, Md., 1974.

Department of Mathematics, Brown University, Providence, Rhode Island 02912 